\title{
NOUVELLE
}

\section{Nécroptose des motoneurones dans des modèles de sclérose latérale amyotrophique familiale ou sporadique}

Virginia Le Verche

> La sclérose latérale amyotrophique (SLA ou maladie de Charcot) est une maladie neurodégénérative de l'adulte qui affecte principalement les motoneurones (MN) supérieurs corticospinaux et/ou les motoneurones inférieurs innervant les muscles squelettiques. La SLA se manifeste par une paralysie progressive accompagnée d'une atrophie musculaire entraînant rapidement la mort lorsque la fonction respiratoire est atteinte. Cette maladie incurable se présente dans la majorité des cas de manière sporadique (SLAs), et seuls environ $10 \%$ des patients souffrent d'une forme génétique héréditaire dite familiale (SLAf). Des mutations dans le gène codant la superoxyde dismutase 1 (SODI) ont été identifiées dans environ $20 \%$ des formes de SLA familiale [10]. Cette découverte a permis le développement de rongeurs transgéniques surexprimant des formes mutantes de cette protéine humaine et présentant les signes caractéristiques de la maladie. Ce modèle animal a été largement utilisé afin d'identifier les mécanismes cellulaires et moléculaires responsables de la mort des motoneurones dans la SLA familiale. De multiples études in vitro et in vivo indiquent que les cellules gliales à proximité des motoneurones jouent un rôle critique dans la neurodégénérescence liée aux mutations de SOD1 [1]. Notre équipe a ainsi précédemment montré que des motoneurones isolés de souris saines meurent spontanément lorsqu'ils sont exposés in vitro à des astrocytes provenant de souris transgéniques porteuses de mutations de SODI telles que SOD1 G93A [2]. Cette mort est due à la libération d'un facteur astrocytaire toxique qui affecte sélectivement les motoneurones et non les autres types neuronaux spinaux présents dans la culture.

Nous avons récemment voulu déterminer si ce phénomène de toxicité produit par les astrocytes murins surexprimant SOD $1^{\text {G93A }}$ pouvait également avoir lieu en présence d'astrocytes provenant de patients souffrant de SLA sporadique. $\varepsilon n$ effet, la présentation clinique des patients affectés par la forme familiale ou sporadique de la maladie est identique, suggérant que ces deux formes partagent certains mécanismes pathologiques.

\section{Développement du premier modèle entièrement humanisé de SLA sporadique}

Dans notre récente étude, nous avons cultivé des astrocytes humains primaires issus du cortex moteur ou de la moelle épinière de patients décédés de SLA sporadique [3]. Nous avons inclus comme contrôles des astrocytes de sujets sains, ainsi que de patients affectés par la maladie d'Alzheimer ou souffrant de broncho-pneumopathie chronique obstructive (BPCO). Nous avons ensuite cultivé sur ces astrocytes des motoneurones humains sains dérivés de cellules souches embryonnaires (hMN) (Figure 1). Tout comme dans notre précédent modèle murin de SLA familiale, nous avons observé que le nombre de motoneurones humains
Columbia University medical center, Center for Motor Neuron Biology and Disease, 630 West Street, Physicians and Surgeons Building, Room 4-401, 10032 New York, Ny, États-Unis.

v12237@columbia.edu

décroît de manière significative lorsque ceux-ci sont cultivés en présence d'astrocytes provenant de patients atteints de SLA sporadique, comparé au nombre de motoneurones humains cultivés sur des astrocytes provenant des patients contrôles. Des experiences menées avec le surnageant des cultures astrocytaires donnent des résultats identiques, indiquant que les astrocytes des patients souffrant de SLA sporadique libèrent un facteur toxique ciblant uniquement les motoneurones. Cette toxicité est spécifiquement associée aux astrocytes car d'autres types cellulaires tels que les fibroblastes ne sont pas nocifs.

Les protéines SOD1 et TDP-43 ne contribuent pas à la toxicité des astrocytes de patients souffrant de SLA sporadique

Nous avons ensuite voulu déterminer par quels mécanismes moléculaires les astrocytes provenant de patients atteints de SLA sporadique sont toxiques. Comme plusieurs études suggèrent que le repliement défectueux de la protéine SODl endogène pourrait jouer un rôle dans la SLA sporadique, nous avons tout d'abord voulu déterminer le rôle de cette protéine, bien que non mutée, dans la toxicité des astrocytes de patients atteints de SLA sporadique $[4,5]$. Pour cela nous avons inhibé par ARN interférence l'expression de la protéine SODl dans les astrocytes de ces patients et constaté la persistance de la toxicité de ces astrocytes envers les motoneurones humains. De la même manière, la toxicité des astrocytes 


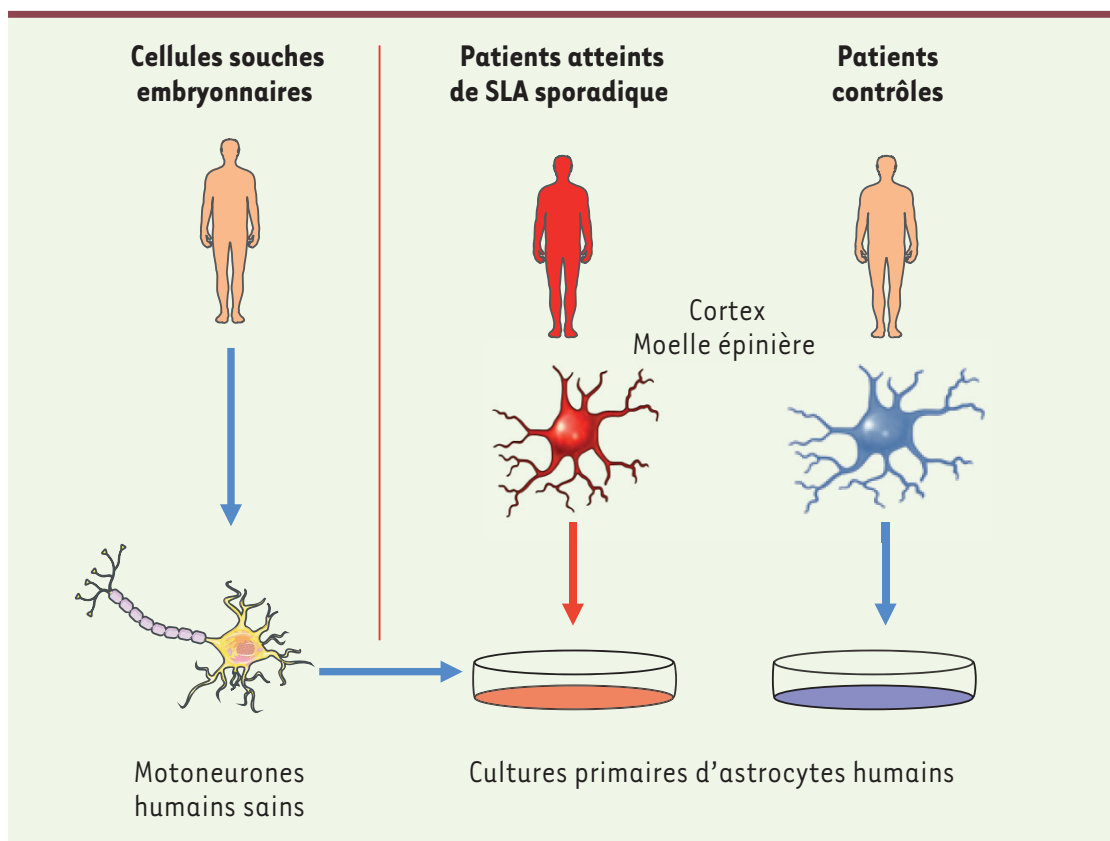

Figure 1. Modèle in vitro de SLA sporadique. Des motoneurones humains sains sont différenciés à partir de cellules souches embryonnaires. Ils sont cultivés sur des astrocytes humains primaires confluents préparés post-mortem à partir de cortex ou de moelle épinière de patients souffrant de SLA sporadique (SLAs) ou de patients contrôles (incluant des patients sains, atteints de maladie d'Alzheimer ou de broncho-pneumopathie chronique obstructive [BPCO]). Après une semaine en coculture, le nombre de motoneurones est compté. Environ $50 \%$ des motoneurones meurent spontanément dans les cocultures en présence d'astrocytes de patients atteints de SLA sporadique, comparé au nombre de motoneurones mourant en présence d'astrocytes contrôles.

envers les motoneurones humains n'est pas abrogée lorsque nous avons inhibé dans les astrocytes l'expression de TAR DNA binding protein 43 (TDP-43), une autre protéine dont des mutations ont été identifiées chez des patients SLA.

\section{Les astrocytes de forme familiale ou sporadique de SLA tuent les motoneurones par nécroptose} Les voies moléculaires menant à la mort cellulaire sont typiquement divisées en deux groupes distincts [11] : I'apoptose, une forme active de mort cellulaire programmée (MCP), est caractérisée par la fragmentation de I'ADN et l'activation de protéases dites caspases. La nécrose est, quant à elle, caractérisée par une rapide perméabilisation membranaire en réponse à des stress extrêmes, sans aucun contrôle moléculaire [6]. Les études histologiques de la moelle épinière de souris transgéniques SODI ${ }^{\text {G93A }}$ ou de patients SLA ne permettent actuellement pas de déterminer de manière certaine laquelle de ces voies est activée dans les motoneurones.

Nous avons observé, dans nos modèles de SLA sporadique et familiale, une augmentation des marqueurs de mort cellulaire programmée dans les motoneurones exposés aux astrocytes toxiques, mais également des signes de perte de la structure membranaire. L'apoptose étant le mode de mort cellulaire programmée le plus répandu, nous avons évalué son implication en traitant les cocultures astrocytes/ motoneurones avec un inhibiteur global des caspases appelé zVADfmk. L'inhibition de l'apoptose n'a eu aucun effet neuroprotecteur, suggérant qu'un autre type de mort cellulaire programmée atypique intervient dans les motoneurones exposés aux astrocytes toxiques.
La nécroptose est une forme de nécrose programmée qui reste peu connue et rarement étudiée dans le système nerveux $[7,11]$. La cascade moléculaire qu'elle met en jeu ne nécessite pas l'activation des caspases, mais fait intervenir des protéines clés telles que les receptor interacting proteins 1 et 3 (RIP1/RIP3) et mixed lineage kinase domain-like (MLKL) [8]. Afin de tester l'implication de cette voie dans nos modèles in vitro de SLA sporadique et familiale, nous avons respectivement: (1) diminué l'expression de RIPl dans les motoneurones par ARN interférence, (2) bloqué dans les motoneurones l'interaction entre RIPl et RIP3 avec une molécule appelée nécrostatine [9], et (3) traité les cocultures humaines avec un inhibiteur de MLKL appelé nécrosulfonamide (NSA). Dans chacune de ces expériences, la mort neuronale a été complètement inhibée, indiquant que les astrocytes toxiques de SLA, qu'il s'agisse de formes familiales ou sporadiques, activent la cascade nécroptotique dans les motoneurones.

\section{Conclusion}

Notre étude montre pour la première fois que les astrocytes primaires provenant de patients souffrant de SLA sporadique expriment la même toxicité envers les motoneurones humains que celle qui est observée dans un modèle murin de SLA familiale. Les astrocytes humains de SLA sporadique et les astrocytes murins porteurs de mutations de SODI libèrent spontanément un ou plusieurs facteurs toxiques qui convergent vers l'activation de la nécroptose sélectivement dans les motoneurones (Figure 2). Ce phénomène n'est pas dépendant des protéines SODI ni TDP-43 dans les astrocytes, mais notre étude n'exclut pas un rôle de ces dernières directement au niveau des motoneurones. Ces travaux pionniers impliquant la nécroptose dans une maladie neurodégénérative ouvrent des voies thérapeutiques prometteuses pour la SLA. De nombreuses questions restent 


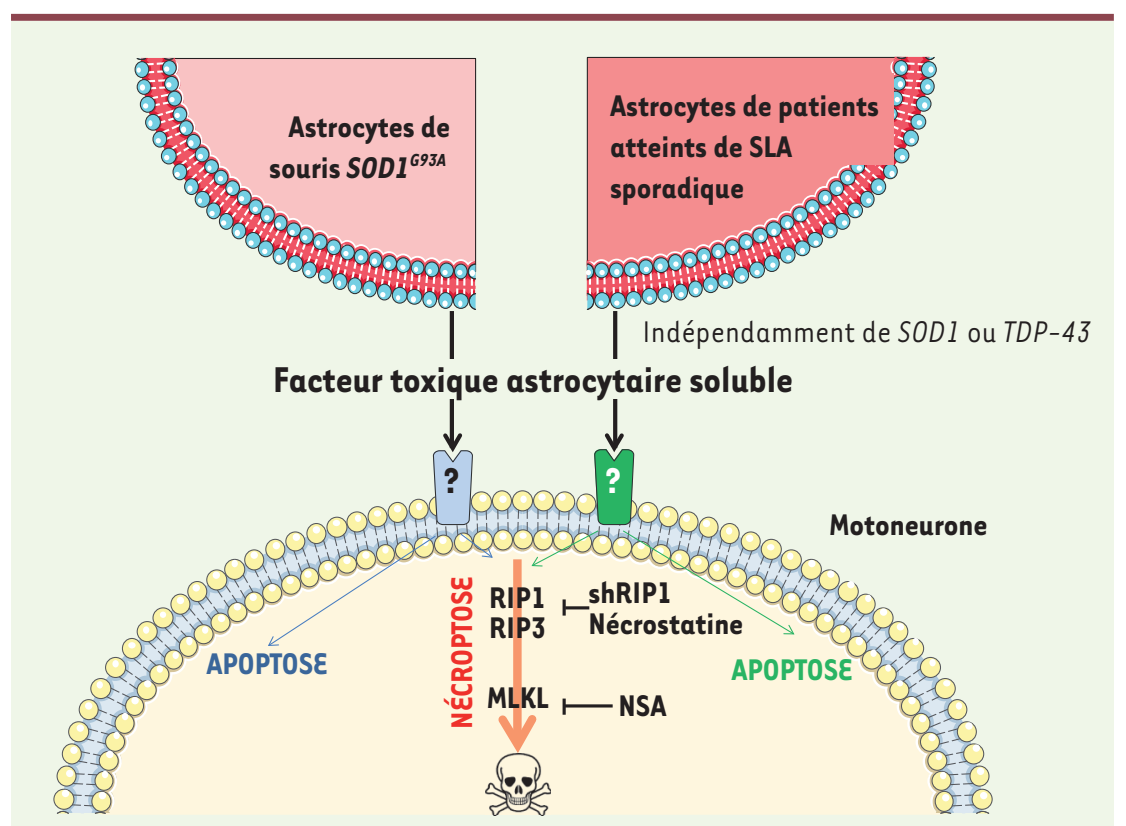

cependant en suspens: comment les astrocytes humains acquièrent-ils leurs propriétés toxiques? Pourquoi les motoneurones sont-ils les seules cellules affectées, et quel est l'élément déclenchant la cascade nécroptotique dans ces neurones? Nous travaillons sur chacune de ces questions afin de mieux comprendre les mécanismes pathologiques de cette maladie incurable. $\diamond$ Familial and sporadic ALS astrocytes activate necroptosis in motor neurons

\section{REMERCIEMENTS}

Les figures ont été créées à partir de la section d'Art Médical du site www.servier.com (http:// creativecommons.org/licenses/by/3.0/).

\section{LIENS D'INTÉRÊT}

Les auteurs déclarent n'avoir aucun lien d'intérêt concernant les données publiées dans cet article.

\section{RÉFÉRENCES}

1. Ilieva H, M. Polymenidou M, Cleveland DW. Non-cell autonomous toxicity in neurodegenerative disorders: ALS and beyond. J Cell Biol 2009 ; 187 : 761-72.

2. Nagai M, Re DB, T. Nagata T, et al. Astrocytes expressing ALS-linked mutated SODI release factors selectively toxic to motor neurons. Nat Neurosci $2007 ; 10: 615-22$.

3. Re DB, Le Verche V, YuC, et al. Necroptosis drives motor neuron death in models of both sporadic and familial ALS. Neuron 2014 ; 81 : 1001-8.

4. Bosco DA, Morfini G, Karabacak NM, et al. Wild-type and mutant SODl share an aberrant conformation and a common pathogenic pathway in ALS. Nat Neurosci 2010 ; 13 : 1396-403.
Figure 2. Mécanismes de mort dans les motoneurones exposés à des astrocytes de SLA familiale ou sporadique. Les astrocytes de souris transgéniques surexprimant SODI ${ }^{\text {G93A }}$ ou de patients décédés de SLA sporadique libèrent un ou plusieurs facteurs toxiques qui produisent des signes mineurs d'apoptose et activent principalement la nécroptose dans les motoneurones. Cette toxicité est indépendante de la présence de SODI ou de TDP-43 dans les astrocytes humains. La nécroptose peut être inhibée en bloquant la formation du complexe RIP1/RIP3 avec la drogue nécrostatine, ou en utilisant des petits ARN en épingle à cheveux dirigés contre RIPl (short hairpin, shRIPl). Enfin, la drogue nécrosulfonamide (NSA) protège également les motoneurones humains en bloquant le facteur MLKL.

5. Guareschi S, Cova $\varepsilon$, Cereda C, et al. An over-oxidized form of superoxide dismutase found in sporadic amyotrophic lateral sclerosis with bulbar onset shares a toxic mechanism with mutant SODl. Proc Natl Acad Sci USA 2012 ; 109 : 5074-9.

6. Vanden Berghe T, Grootjans S, Goossens V, et al. Determination of apoptotic and necrotic cell death in vitro and in vivo. Methods 2013 ; 61 : 117-29.

7. Vandenabeele P, Galluzzi L, Vanden Berghe T, Kroemer G. Molecular mechanisms of necroptosis: an ordered cellular explosion. Nat Rev Mol Cell Biol 2010 ; 11 : 700-14.

8. Moujalled DM, Cook WD, Murphy JM, Vaux DL. Necroptosis induced by RIPK3 requires MLKL but not Drpl. Cell Death Dis 2014 ; 5 : el086.

9. Degterev A, Hitomi J, Germscheid M, et al. Identification of RIPl kinase as a specific cellular target of necrostatins. Nat Chem Biol 2008 ; 4 : 313-21.

10. Dupuis L, Loeffler JP. Sclérose latérale amyotrophique, jonction neuromusculaire et déficit énergétique. Med Sci (Paris) 2008 ; $24: 1077-82$.

11. Cabon L, Martinez-Torres, Susin SA. La mort cellulaire programmée ne manque pas de vocabulaire. Med Sci (Paris) $2013 ; 29: 1117-24$

\begin{tabular}{|c|c|}
\hline & Bon de commande \\
\hline 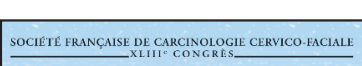 & $\begin{array}{l}\text { À retourner à EDK, 109, avenue Aristide Briand - } 92541 \text { Montrouge Cedex } \\
\text { Tél. : } 0141177405 \text { - Fax : } 0143293262 \text { - E-mail : edk@edk.fr }\end{array}$ \\
\hline \multirow{10}{*}{$\begin{array}{l}\text { Limagerie dans la prise } \\
\text { en charge des cancers des voies } \\
\text { aérodigestives supérieures }\end{array}$} & NOM : $\ldots$ Prénom : \\
\hline & Adresse : \\
\hline & Code postal : $\quad$ Ville : \\
\hline & Pays : ........................ \\
\hline & Fonction : \\
\hline & $\begin{array}{l}\text { Je souhaite recevoir l'ouvrage L'imagerie dans la prise en charge des cancers des voies aérodigestives } \\
\text { supérieures : } 35 €+3 € \text { de port }=\mathbf{3 8} € \text { TTC }\end{array}$ \\
\hline & en ................ exemplaire, soit un total de ................................ $€$ \\
\hline & $\square$ Par chèque, à l'ordre de $\mathbf{E} \mathbf{D} \mathbf{K}$ \\
\hline & $\square$ Par carte bancaire : $\quad \square$ Visa \\
\hline & 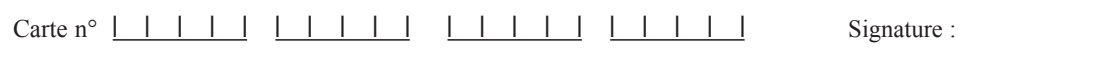 \\
\hline & $\mathrm{N}^{\circ}$ de contrôle au dos de la carte : $\quad|\quad| \quad \mid$ \\
\hline
\end{tabular}

